Voix et Images

voixetimages

\title{
Parcours emblématiques : Languirand et Ronfard
}

\section{Lucie Robert}

Volume 29, numéro 1 (85), automne 2003

\section{Claire Martin}

URI : https://id.erudit.org/iderudit/007547ar

DOI : https://doi.org/10.7202/007547ar

Aller au sommaire du numéro

Éditeur(s)

Université du Québec à Montréal

ISSN

0318-9201 (imprimé)

1705-933X (numérique)

Découvrir la revue

Citer ce compte rendu

Robert, L. (2003). Compte rendu de [Parcours emblématiques : Languirand et Ronfard]. Voix et Images, 29(1), 149-154. https://doi.org/10.7202/007547ar d'utilisation que vous pouvez consulter en ligne.

https://apropos.erudit.org/fr/usagers/politique-dutilisation/ 


\section{R A M A T U R G I E}

Parcours emblématiques: Languirand et Ronfard

$$
+++
$$

\section{LUCIE ROBERT}

Université du Québec à Montréal

Quand sonne l'heure des bilans, rares sont ceux qui ont le courage d'affronter leur parcours et de le soumettre une seconde fois au jugement des générations plus jeunes. Sans doute faut-il voir là la volonté de laisser un héritage ou du moins un témoignage de ces longues carrières. Cette heure a vraisemblablement sonné pour Jacques Languirand et Jean-Pierre Ronfard qui, ayant passé le cap des soixante-dix ans l'un et l'autre, publient non pas des œuvres complètes, mais quelque chose qui se rapproche de ce qu'on appelle, dans l'industrie du disque, des «compilations». Ainsi, après avoir renoncé au thêâtre au profit de la carrière radiophonique que l'on sait, Jacques Languirand réédite son théâtre, en intégrale, sous le titre Presque tout Languirand ${ }^{1}$ (il manque à l'œuvre complète son roman, Tout compte fait, son Dictionnaire insolite et ses divers essais). Publiée en 2001, cette réédition du théâtre précédait de peu l'annonce d'une production en français de Man Inc., près de vingt ans après sa création en anglais dans une traduction de Mavor Moore. Ce fut chose faite au printemps 2003. Quant à JeanPierre Ronfard, il publie aussi, en deux volumes, ses Écritures dramatiques ${ }^{2}$, dans lesquelles il faut aussi voir la mémoire de Robert Gravel, son proche collaborateur. Peutêtre d'ailleurs les publie-t-il plus en hommage à celui-ci qu'en mémoire de son œuvre à lui.

Toutefois, loin d'en rester au devoir de mémoire, Languirand et Ronfard ajoutent à ces sortes d'anthologies des œuvres plus récentes, publiées à part, comme autant de marques de leur activité présente. Il ne saurait s'agir d'une simple coïncidence puisque l'un et l'autre ont, au cours de leur carrière, été plutôt chiches en matière d'édition. Languirand, qui n'a jamais été aussi jeune, en remet avec une toute nouvelle pièce, Faust et les radicaux libres $^{3}$, alors que Jean-Pierre Ronfard édite les deux pièces qu'il a écrites avec Alexis Martin, Transit section $n^{\circ} 20$ et Hitler ${ }^{4}$. 
J'ai toujours été séduite par la fraîcheur qui se dégage des premiers textes dramatiques de Jacques Languirand. Ils ont, bien sûr, un peu vieilli dans leur style et leur objet, qui empruntaient peut-être un peu trop facilement aux grands classiques de la dramaturgie d'avant-garde: l'influence marquée de Ionesco, Beckett ou Adamov se lit aisément à travers Les insolites, Le roi ivre, Les grands départs, Les violons de l'automne ou Le gibet. Le dérèglement d'une action dramatique remarquablement simple, portée par des personnages fragiles, perdus dans un monde qui les déroute, conserve toutefois l'attrait de ces œuvres qui, produites dans la grisaille des années cinquante, posent les questions les plus fondamentales de la condition humaine. Euvre de jeunesse, l'œuvre dramatique de Languirand l'est certainement, et c'est en premier lieu ce qui lui confère à la fois sa fraîcheur et son immense naïveté. Dépassée, l'œuvre dramatique de Languirand l'est tout autant, comme la plupart de ses modèles, mais bien coupable serait celui ou celle qui négligerait de voir là une œuvre significative.

Plus tardives, et moins connues, sont Les cloisons, Klondyke et Man Inc., celle-ci étant jusqu'à présent restée inédite. Cette série, rédigée dans les années soixante, marque l'abandon progressif des modèles proposés par le thêâtre d'avant-garde français et l'intégration progressive d'une approche multimédiatique. Les cloisons (1962) n'a été créée à Washington qu'en mai 2001. La pièce se présente comme une sorte de dialogue entre deux amoureux à travers une cloison. Lui est déjà dans la chambre d’hôtel quand elle arrive dans la sienne, à la suite d'une rupture amoureuse. Bruits de pas, de robinets, de fenêtres rythment les monologues parallèles de deux personnages qui envisagent avec espoir et hésitation le début d'une relation amoureuse avec l'Autre. Klondyke (1965) est rééditée ici sans sa préface, «Le Québec et l'américanité», qui avait fait date à l'époque, étant reçue comme le premier grand énoncé de réflexion sur le sujet. La pièce n'avait pas connu un grand succès au moment de sa création, sans doute un peu par la faute de l'auteur. Cette histoire de chercheurs d'or est, en effet, désespérément bavarde, mais elle répond à un registre radicalement différent des précédentes par le recours aux principes du théâtre épique. Le changement était majeur dans l'œuvre de Languirand, du moins au plan formel, puisqu'il entraînait la conception d'un théâtre plus complexe, où étaient conviées la musique et la chanson, utilisées ici comme des songs brechtiens. Man Inc. (1967) forme un spectacle qui allie la musique, le thêâtre, la danse et le cinéma. L'auteur revient au mythe de Prométhée, mais aussi à la Genèse puis à l'Apocalypse. Nous serions encore à l'âge de pierre d'une civilisation harmonieuse, à nous entourer d'objets de consommation, oubliant les valeurs authentiques et tranquilles qui donnent sens à la vie. La pièce oppose Pierre, le premier homme, et Madeleine, la première femme. Ils sont mariés, vivent en banlieue, ont un enfant et en attendent un autre, mais ils rêvent d'autre chose.

Fasciné par les thèses d'Herbert Marcuse, passionné par le développement des moyens de communication, Languirand devait passer les trente années suivantes à déployer sa faconde sur les ondes de Radio-Canada, au profit de plusieurs générations successives d'auditeurs. Voilà qu'il en remet dans une pièce qui n'est pas si loin de la dramaturgie proposée dans Man Inc. Dédiée à Jan Doat, son «maître de théâtre», Faust a obtenu le prix spécial du jury de la fondation Alexandre S. Onassis en 2001, attribué pour la première fois à une pièce en langue française. Dans un mot écrit pour la création avortée de Man Inc. en 1970, l'auteur inscrit sa démarche dans le projet de créer une «grammaire de la communication». (Presque tout Languirand, 805) Il précise : «En principe, dans les multimédia, chaque médium doit véhiculer son propre contenu. Il naît 
alors du rapport entre les deux contenus, ou les deux messages, un troisième contenu ou message total qui est toujours plus grand que la somme des deux parties.» (Ibid.) Mise en lecture le 16 octobre 2002 à Québec, au Théâtre Niveau Parking, dans le cadre des Rendez-vous dramatiques, sous la direction de Mathieu Gaumond, la pièce aurait été écrite à la suggestion de Robert Lepage, au moment où Languirand collaborait à la création d'un de ses spectacles. L'esthétique de Languirand, qui s'est toujours intéressé à la communication, est proche de celle de Lepage en ce qu'elle repose sur la création d'un espace scénique multimédiatique, opposant les technologies de l'écran à l'univers de la scène vivante. La scénographie de Faust mélange ainsi le classique et le postmoderne, le cinéma de série B (les films d'horreur, Blade Runner), la télévision (Star Trek: The Next Generation est évoqué à plusieurs reprises), les reposoirs de la Fête-Dieu et la technologie actuelle.

Il n'est peut-être pas inutile de souligner l'intérêt qu'a retrouvé le personnage de Faust auprès des écrivains contemporains. Languirand rejoint ici Robert Léger ${ }^{5}$, en reprenant le personnage pour discuter des conditions de la recherche scientifique, des nouvelles technologies de reproduction et, en particulier, du clonage, qui, dans le cas présent, se superpose au rêve d'un homme-machine, d'un cyborg. C'est cependant un Faust vieillissant qui rencontre Lucifer, un élégant jeune dandy, qui accepte de «reculer les horloges biologiques» (27) et de garantir l'éternelle jeunesse. Il retient «une jeune femme au moment où elle va rendre l'âme» (37), donnant une seconde vie à Marguerite. Personnage déroutant, Lucifer ne veut pas de l'âme de Faust, qui ne lui a d'ailleurs rien demandé au départ. Il s'ennuie et cherche à se désennuyer. Cependant, Faust découvre qu'avoir trente ans n'est pas si simple: «Du coup, j'appartiens à une génération qui n'a pas tellement d'ardeur!» (80) Lucifer lui propose alors un projet de couple: la création de l'homme nouveau. On se retrouve devant un chantier qui «évoque celui du docteur Frankenstein» (85) pour produire un enfant qui arrive «avec son modem, son fax, son cellulaire et le tout intégré» (86). Toutefois, l'enfant, Frank, est malheureux : il voudrait être utile, faire quelque chose de ses mains, avoir des amis, des intimes, une compagne. Un soir, il a cassé le cou d'une jeune femme qui pleurait dans le parc, et il songe désormais à mettre fin à ses jours. Victime de cette "crise d'adolescence», la famille est devenue dysfonctionnelle. Faust n'est cependant pas au bout de ses peines, car l'Inquisiteur, venu évaluer les dégâts, condamne Lucifer à «l'humanitude» (169), c'est-à-dire à "penser dans le temps, [...] agir dans le temps et même [...] vivre dans le temps» (167). Celui-ci ne s'en plaint guère et l'on apprend bientôt que Marguerite est enceinte. "Lulu» sera donc le fils de Faust, vieux et mourant: "Grâce à toi, Henry, se réjouit Lucifer, j'ai échangé une condamnation à errer sans fin dans l'éternité pour une condamnation à errer dans le temps.» (191)

Dans la présentation de ses Écritures dramatiques, Jean-Pierre Ronfard explique: «J'ai toujours considéré que je n'écrivais pas pour l'éternité, que l'écriture dramatique est une chose destinée à la réalisation, que donc elle ne devrait avoir que les caractéristiques du réel: utile, transformable et éphémère. C'est pourquoi ces pièces ayant été jouées,

$+++$

5 Robert Léger, Faust. Chronique de la démesure, Ottawa, Le Nordir, 2001, 165 p. 
c'est-à-dire ayant rempli leur fonction, elles sont restées longtemps enfouies dans des tiroirs profonds.» (5) Enfouies, mais non perdues, on le notera, du moins pas toutes. La collection de pièces qu'il publie couvre son activité au Nouveau Thêâtre expérimental entre 1986 et 1998. Il s'agit donc de la période postérieure à la création de Vie et mort du Roi boiteux (1983), une période dont nous ne connaissions, sous forme de livre, que les Cinq études ${ }^{6}$. La plupart des pièces qui forment ces Écritures dramatiques représentent le résultat d'un travail collectif: elles ont été conçues avec Robert Gravel, parfois avec Anne-Marie Provencher.

Sommes-nous dans l'ordre de la création collective rédigée après coup? Sans doute parfois. Nous sommes surtout dans l'ordre de la mémoire. En quatrième de couverture, l'auteur écrit: «il est possible que des textes, écrits dans le seul but de la représentation immédiate, prennent une nouvelle vie grâce à la publication ». Aussi les didascalies qui accompagnent les textes sont-elles toutes rédigées à l'imparfait, plutôt qu'au présent, comme le commandent depuis la Renaissance les règles de l'édition théâtrale: «les didascalies sont ici pour rappeler ce qui s'est passé, non pour donner des ordres à de futurs réalisateurs» (5-6). C'est là nier la légitimité de l'activité de lecture au thêâtre, car la didascalie, quoi qu'on en dise par ailleurs, n'est jamais vraiment un ordre ni un impératif - aucun metteur en scène ne s'y sent contraint. Elle est, en revanche, une manière de favoriser la lecture. En ce cas, les didascalies au passé ne donnent à lire, sans imagination, qu'un spectacle réalisé alors que le présent permet au lecteur de se propulser dans un monde imaginaire qu'il a créé lui-même de toutes pièces.

Le choix est malheureux, car certains des textes dramatiques édités ici méritent d'être relus comme des pièces de théâtre. C'est le cas du Grand théâtre du monde/El gran teatro del mundo (1989), écrite d'après Calderón, qui réactualise le personnage de Don Juan et multiplie les plans de réalité (au présent et au passé, ici et en Espagne) pour mieux rendre compte de la proposition de l'auteur espagnol, pour qui le "grand thêâtre du monde» se joue d'abord dans les coulisses. C'est le cas aussi, mais sur un registre différent, du Précis d'histoire générale du théâtre en 114 minutes (1992), structuré comme une représentation unique, mais à l'envers. La pièce s'ouvre sur le salut des acteurs au public et se termine sur le dévoilement de la scénographie et les premières répétitions. À rebours sera donc aussi contée l'histoire, de Brecht à Euripide. À la répétition sont réunis quelques personnages représentatifs de cette histoire: Faust, Ubu, Julie, Madame Lauzon, Romain, Violette et Olga, la Célestine. Les scènes sont coupées par les «proférateurs» qui citent des extraits d'un ouvrage sur le théâtre. On a évidemment choisi les phrases grandiloquentes et vides pour créer une opposition facile entre «faire» et "parler de». Enfin, on notera Matines. Sade au petit déjeuner (1996), pièce dont on se rappellera peut-être qu'elle avait causé un certain émoi, non à cause de son sujet, mais à cause de l'heure de la représentation: 7 h 30, le matin, avec café et croissants. La pièce réunit cinq personnages, quatre acteurs et un régisseur, qui doivent enregistrer une adaptation de La philosophie dans le boudoir du marquis de Sade pour la radio. Le double plan de jeu - pendant que certains acteurs jouent pour la radio, d'autres discutent de Sade - permet d'entrecouper les scènes érotiques de discussions philosophiques et politiques, activant ainsi simultanément les trois isotopies fondamentales de l'œuvre du marquis.

\footnotetext{
6 Jean-Pierre Ronfard, Cinq études, Montréal, Leméac, coll. «Thêâtre», 1994, 134 p.
} 
À Beloeil ou ailleurs (1986) n'aurait pas non plus manqué d'intérêt pour le lecteur si les 40 brefs scénarios avaient été conservés. Dans cette création collective, sept personnages portant les mêmes noms se produisent en 40 scénarios de pièces affichés au mur et dont l'action se situe sur quatre siècles. À chaque représentation, deux ou trois scénarios étaient joués et donc biffés. Ne restent que quelques pièces rescapées de l'aventure, assez pour saisir le projet d'ensemble, trop peu pour en évaluer les résultats. Tête à tête (1994) est «une tentative de faire entrer un public à l'intérieur de cette étrange démarche, confuse et implacable, qu'est le désir de la création à deux, mêlé au désir assez puéril mais réel de se démarquer des autres créateurs de théâtre» (tome II, 9). La pièce oppose ainsi les personnages de Gilles et de Jean-Patrice au moment où ils discutent de la conception de leur prochain spectacle, qu'ils veulent inspiré du thêâtre élisabéthain. Le projet initial était de monter Le Juif de Malte de Christopher Marlowe. À l'arrivée, JeanPatrice a écrit une pièce de son cru, Histoire de la gloire et de la décadence d'Empédocle d'Agrigente. L'ensemble offre un point de vue qui ne manque pas d'intérêt sur le processus même de la création au NTE, mais qui n'a évidemment pas l'intérêt dramatique des "grandes» pièces. Enfin, Cinquante (1995), conçue et mise en scène avec Robert Gravel, se présente comme la réalisation du projet qui clôt Tête à tête et qui est plus un exercice de style quautre chose: 50 comédiens sont en scène, 25 hommes et 25 femmes, qui interprètent 50 brèves scènes différentes, reconstitutions de tableaux célèbres, discours, situations variées. Enfin, les trois pièces qui forment le dernier volume sont ultérieures au travail de Robert Gravel, qui avait néanmoins collaboré à la première, Le cru et le cuit (1995), dont nous n'avons ici qu'un fragment, soit le monologue initial. En onze tableaux toutes les deux, Les amours (1997) explore diverses facettes du thème éponyme alors que Les mots (1998), conçue avec la graphiste Sylvie Daigle, se présente comme une nouvelle "étude thêâtrale», c'est-à-dire comme une réflexion sur une des composantes essentielles du spectacle théâtral. Ni l'une ni l'autre n'offre l'intérêt des pièces précédentes et elles sont plus des documents que des oeuvres.

Autant les pièces conçues avec Robert Gravel et Anne-Marie Provencher soulèvent la question même du thêâtre, opposant ce qu'il est profondément aux traditions qui l'ont à la fois construit et perverti, autant les deux pièces écrites avec Alexis Martin se situent ailleurs. Il ne s'agit plus de rendre le médium apparent en explorant toutes ses contraintes, mais de réfléchir à la manière dont l'écriture dramatique peut rendre compte du réel. Transit no 20 et Hitler sont ainsi deux pièces qui posent le problème de l'Histoire. Dans sa Théorie du drame moderne, Peter Szondi rappelait justement combien le drame était hostile à l'Histoire, comment le caractère à la fois organique et synthétique du thêâtre dramatique ne permet guère d'explorer l'Histoire autrement qu'en en mesurant les conséquences sur l'individu ${ }^{7}$. Et si le thêâtre épique a pu rétablir avec l'Histoire une relation féconde, c'est en introduisant, à la manière de l'épopée, un sens à cette Histoire, une finalité du présent projetée vers l'avenir. Aussi les sujets du thêâtre épique sont-ils souvent empruntés au passé, c'est-à-dire à un temps clos, un temps dont les effets nous

$+++$

7 Peter Szondi, Théorie du drame moderne, 1880-1950, trad. de l'allemand par Patrice Pavis, avec la collaboration de Jean et Mayotte Bollack, Lausanne, L'Âge d'homme, 1983. 
sont connus. Tel n'est pas le cas ici, où l'écriture tente l'expérience de l'histoire contemporaine. En fait, écrivent les auteurs, «Histoire et théâtre, malgré leur attraction réciproque, sont par nature bien différents. Le but de l'Histoire est d'établir la vérité à l'aide de documents indubitables. [...] Le but du thêâtre est d'émouvoir un public par une fiction qui ait les allures de la vérité. [...] Le thêâtre historique tente de conjuguer ces deux forces parallèles égales tirant en sens contraire (ce qui, en physique, définit la dynamique des couples!).» (6-7)

Transit $n^{\circ} 20$ a été créée le $1^{\text {er }}$ février 2000 à l'Espace libre, dans une production du Nouveau Théâtre expérimental mise en scène par Alexis Martin. La pièce s'ouvre sur un tableau intitulé «Enterrement de Nietzsche» et sur deux personnages, Lanoix et Legrand, «cols bleus, concierges, appariteurs, bruiteurs, hommes à tout faire de l'Histoire» (13). On apprendra qu'ils gèrent surtout le conteneur du transit, section 20, soit les poubelles de l'histoire du vingtième siècle: "un transit c'est comme qui dirait un passage, ou plus exactement, tout ce qui se passe entre deux bornes fixes, le début et la fin. Et puis, l'aire de transit, c'est le lieu où tout ça arrive. Et l'histoire [...] c'est le souvenir qu'on garde de tout ce qui est arrivé pendant le transit, de tout ce que l'humanité y a vécu.» (122) En dixsept tableaux est ainsi relatée une histoire du vingtième siècle, qui superpose les événements de même nature (le troisième tableau se passe indifféremment en Grèce en 1921, en Allemagne en 1945, en Ukraine en 1941, en Espagne en 1936 ou en Bosnie en 1996) et les personnages en apparence opposés (Maïakovski et Leni Riefenstahl, le journaliste de la Pravda et le soldat de la CIA, Anne Frank et le soldat François Rochette, deux victimes de guerre qui essaient d'imaginer l'avenir qu'ils auraient pu avoir).

«Quand y a pas de documents... On peut seulement imaginer!», écrivent encore les auteurs (33). Illustrant ce principe, Hitler a été créée à l'Espace libre dans une production du Nouveau Théâtre expérimental, le 6 février 2001, dans une mise en scène des auteurs. La pièce se divise en huit épisodes qui se déroulent en avril 1945, dans le Bunker de Berlin. Ayant choisi un moment dont, précisément, nous ne savons pas grand-chose, les auteurs ont toute liberté pour créer un personnage mégalomane, grandiloquent, maniaque et misogyne. Au milieu du septième épisode, la fiction s'interrompt brutalement: Alexis, l'auteur-acteur, n'en peut plus. La rupture du jeu met en valeur le travail d'écriture, en particulier le travail de construction linguistique, que l'on ne remarquerait peut-être pas autrement: «Hitler, c'était un orateur. La langue, c'est primordial. Ça nous prenait une langue spécifique pour un objet spécifique.» (191) La pièce présente ainsi une sonorité particulière, dans une langue, d'où sont, autant que possible, éliminées les voyelles, surtout les nasales, ce qui «va contre l'esprit du français» (190). Après cette mise au point, la pièce reprend son déroulement: après avoir tué Eva Braun, Hitler assassine son sosie, met le feu au Bunker et se sauve.

Dans une postface intitulée "Lacunes, fiction, histoire», les auteurs constatent que «c'est dans les blancs de l'histoire, ses trous, que le roman du théâtre peut naître» (205) puisqu'ils permettent de «brode[r] avec un sans-gêne épouvantable» (206). C'est dans ce registre qu'opère Hitler, alors que, à l'inverse, Transit $n^{\circ} 20$ conserve la mémoire d'événements réels qui ont marqué le siècle. Aussi les deux pièces diffèrent-elles radicalement dans leur écriture, bien qu'elles soient portées par un même projet qui, en définitive, est celui de réfléchir au «destin de la parole au vingtième siècle» (207). Aux paroles perdues et aux paroles oubliées, surtout. Et l'on remerciera Jean-Pierre Ronfard d'avoir au contraire, comme Languirand, assuré la mémoire de la sienne propre, en permettant à sa parole d'errer dans le temps plutôt que dans l'éternité. 